\title{
Management of unilateral staghorn renal stones with concurrent urinary tract infections by retroperitoneal laparoscopic pyelolithotomy with prolonged renal posterior lower segment incision
}

\author{
BOWEN SHI ${ }^{*}, \mathrm{XI} \mathrm{HONG}{ }^{*}$ and JIANJUN YU \\ Department of Urology, The Sixth People's Hospital Affiliated to Shanghai Jiao Tong University, \\ Shanghai 200233, P.R. China
}

Received October 13, 2018; Accepted April 5, 2019

DOI: $10.3892 /$ etm.2019.7545

\begin{abstract}
The present study evaluated the effectiveness and safety of the removal of unilateral staghorn renal stones with concurrent infections by retroperitoneal laparoscopic pyelolithotomy (RLP) with prolonged renal posterior lower segment incision. Patients with staghorn renal stone and concurrent urinary tract infection (UTI) who underwent RLP with prolonged renal posterior lower segment incision as the primary, one-session treatment at our institution between March 2014 and December 2017 were retrospectively reviewed. Routine laboratory tests were performed and the patients received broad-spectrum intravenous antibiotics from at least 3 days prior to the operation. All patients were examined pre-operatively by urinary ultrasonography, computed tomography or intravenous urography. UTI was confirmed by laboratory tests with or without radiographic evidence by an experienced urologist. All patients (18 females and 10 males) successfully underwent the procedures and there was no conversion to open surgery in any case. The mean age was 57.0 \pm 10.81 years (age range, 40-74 years) and the mean calculus size was $3.3 \pm 0.79 \mathrm{~cm}$. The mean operation time, warm ischemia time and post-operative hospital stay were $114.4 \pm 12.09 \mathrm{~min}, 28.1 \pm 4.23 \mathrm{~min}$ and $5.8 \pm 1.42$ days, respectively. The mean hemoglobin drop on day 3 following surgery was $0.5 \pm 0.38 \mathrm{~g} / \mathrm{dl}$ and there was no requirement for blood transfusion in any patient. The mean change of serum creatinine levels between pre-operative baseline and post-operative day 3 or post-operative month 6 was $6.0 \pm 20.03$
\end{abstract}

Correspondence to: Dr Jianjun Yu, Department of Urology, The Sixth People's Hospital Affiliated to Shanghai Jiao Tong University, 600 Yishan Road, Xuhui, Shanghai 200233, P.R. China

E-mail: ycx917@126.com

${ }^{*}$ Contributed equally

Key words: staghorn renal stone, retroperitoneal laparoscopic pyelolithotomy, nephrolithotomy, urinary tract infections or $-4.5 \pm 15.13 \mu \mathrm{mol} / 1$, respectively. The stone-free rate was $100 \%$ at 3 days and at 6 months. Mild post-operative complications (Grade I or II) occurred in 6 patients, including temporary and constant elevated body temperature $\left(>38.5^{\circ} \mathrm{C}\right)$. No severe complications, including urine leakage, sepsis, residual stones requiring auxiliary procedures, were noted and there were no circumstances requiring further surgical intervention in any of the patients. In conclusion, RLP with prolonged renal posterior lower segment incision is an effective and safe procedure for patients with staghorn renal stones and concurrent UTI, and its feasible application as a single-session monotherapy is particularly convenient considering the financial and medical situation, as well as the patients' preference.

\section{Introduction}

The management of staghorn renal stones remains a challenge in urology. The success rates and widespread application of endourological, ureteroscopic and percutaneous techniques have limited the open surgical nephrolithotomy to cases with complex staghorn calculi (1). As recommended in the 2005 and 2016 American Urological Association (AUA) guidelines, percutaneous nephrolithotomy (PCNL) should be the first-line treatment for most patients with staghorn calculi $(2,3)$. According to the European Association of Urology (EAU) guidelines, most complex stones, including partial and complete staghorn stones, should be approached primarily with PCNL (4). However, if percutaneous approaches are not likely to be successful, or if multiple endourological approaches have been performed unsuccessfully; open or laparoscopic surgery may be a treatment option (4). Although PCNL is a minimally invasive procedure with a comparatively higher stone-free rate (SFR), it bears a considerable risk of serious complications, including severe bleeding and post-operative sepsis (5).

During PCNL for staghorn calculi, blood loss is significantly increased compared with that occurring in this procedure for other types of stone (6). Urosepsis after upper urinary tract endoscopic surgery is a life-threatening complication and considered to be associated with high intrapelvic pressure and large stone size (7). In addition, it remains controversial which procedures are best for patients with staghorn renal stone and 
concurrent urinary tract infection (UTI). Single-session PCNL is normally selected for large renal stones with infections due to high risk of post-operative sepsis, and surgical decompression (drainage of the kidney) is more crucial for life-saving procedures in obstructed urinary tracts (8).

The ideal procedure for large or staghorn renal stones should achieve a complete stone-free status with minimal morbidity and with the least number of procedures (5). Therefore, laparoscopic pyelolithotomy (LP) is an eligible monotherapy for complex staghorn renal stone with UTI. At our institution, retroperitoneal LP (RLP) is performed as a routine procedure due to its advantages of avoiding contamination or damage to the abdominal organs. It has been demonstrated that RLP is safe and effective for large renal stones (9), yet the indications for RLP have not been defined. In addition, laparoscopic anatrophic nephrolithotomy (LAN) is an alternative treatment modality for complete staghorn renal stone, but it may cause a minimal loss of function in the affected kidney (10). For better exposure of vision and complete clearance of stones, a prolonged renal posterior lower segment incision is performed during RLP as a single-session management (11). The present study reports on the experience at our center in the management of unilateral staghorn renal stones with infections by RLP with prolonged renal posterior lower segment incision, and evaluated the effectiveness and outcomes.

\section{Patients and methods}

Patients. The present retrospective study included a total of 28 patients with staghorn renal stone and concurrent UTIs who underwent RLP with prolonged renal posterior lower segment incision as a primary, one-session treatment at the Sixth People's Hospital affiliated to Shanghai Jiao Tong University (Shanghai, China) between March 2014 and December 2017. The inclusion criteria included patients with unilateral staghorn stones and concurrent UTIs, patients' personal preference for decisive single-session treatment and predicted difficulties of stone removal due to the small renal calyx or large stone burden. The exclusion criteria included combined distal ureteral calculi, stone size of $<2 \mathrm{~cm}$, severe obstruction of the urinary tract, history of ipsilateral open renal surgery, solitary kidney (anatomical or functional), concurrent renal stone-associated sepsis or septic shock [according to the criteria for Sepsis-3 (12-14)], surgical contraindications and patients' preference for more minimally invasive procedures.

The patients were fully informed that, according to the EAU and AUA guidelines, the first-line treatment of staghorn stones is PCNL. However, concurrent UTI and large stone burden may require multiple-session PCNL for the clearance of stones. It is unquestionable that PCNL would be a more minimally invasive management than RLP with prolonged renal posterior lower segment incision. The patients were fully informed that warm ischemia time would influence the kidney function to a certain extent. Written informed consent for the operation was provided by each patient prior to the surgery at the time of hospitalization. The Ethics Committee of the Sixth People's Hospital Affiliated to Shanghai Jiao Tong University School of Medicine (Shanghai, China) approved the present retrospective study. The treatment is also in accordance with the Declaration of Helsinki.
Pre-operative assessments and preparation of patients. All patients were examined pre-operatively with urinary ultrasonography (US), computed tomography or intravenous urography (IVU) to evaluate the renal anatomy and stone burden, including size (largest diameter) and distribution (involved renal collecting system). All patients underwent routine laboratory tests, including blood chemistry, complete blood count, urine analysis and urine culture and received broad-spectrum intravenous antibiotics at least 3 days prior to the operation. The UTIs were confirmed by laboratory tests (e.g. as leukocyturia or bacteriuria) with or without radiographic evidence by one experienced urologist at our institution. If the presence of UTIs was uncertain, $\geq 3$ urologists drew a conclusion by discussion. Subsequently, empirical broad-spectrum antibiotic therapy was adopted according to the results of the urine cultures.

Surgical technique. The RLP was performed similarly to previously reported procedures (15-17), except for the incision extending to the renal parenchyma and temporary blockage of the renal artery. The representative images in Figs. 1-4 provide details of the laparoscopic procedure. Patients were under general anesthesia and placed in the lateral decubitus position with a waist bridge. The retroperitoneal space was first separated by using fingers and then expanded by a customized gas balloon (inflated to $\sim 500 \mathrm{ml}$ ) through the first incision at the posterior axillary line under the 12th rib margin. With the guidance of the index finger, two additional operating ports $(5$ and $10 \mathrm{~mm})$ were placed at the anterior axillary under the costal margin and $2 \mathrm{~cm}$ above the iliac crest, respectively. Retroperitoneal fat was carefully separated to spare the space prior to opening the perirenal fascia. The retroperitoneal compartment was revealed and subsequently, the area along the ureter toward the medial margin of the psoas muscle was searched, fully exposing the renal pelvis in the renal sinus (9), and the position of the stone was confirmed. After complete dissection of the renal pedicle, the kidney was mobilized and the renal artery was temporarily clamped with an atraumatic bulldog clamp (18). The incision was along with the direction of the ureter to renal pelvis and expanded to the junction of the renal parenchyma and pelvis. Of note, the diameter of the nephrotomy incision was kept $<2 \mathrm{~cm}$ to as far as possible minimize the renal function loss after the operation. After removing the stone, a pediatric catheter was placed to flush out any residual stones and pus with normal saline until the rinsing solution became clear. On laparoscopy, all renal calyces were visible clearly under the camera's lens through the dilated renal pelvis, allowing for the identification of any possible existing residual stones. After confirming no active bleeding, the renal parenchyma was sutured continuously with 2-0 absorbable sutures. After the clamp of the renal artery was released, renal perfusion was confirmed and a check for any bleeding sites from the nephrotomy incision was performed (18). After insertion of the double-J ureteral stent (Fr6), the renal pelvis was sutured with 4-0 absorbable sutures. In addition, a suction drainage tube was indwelled in the peripelvic tissues. After the surgery, the volume of wound drainage fluid was monitored and recorded every day. The patients were considered to have urine leakage if the following situation occurs: Increased 


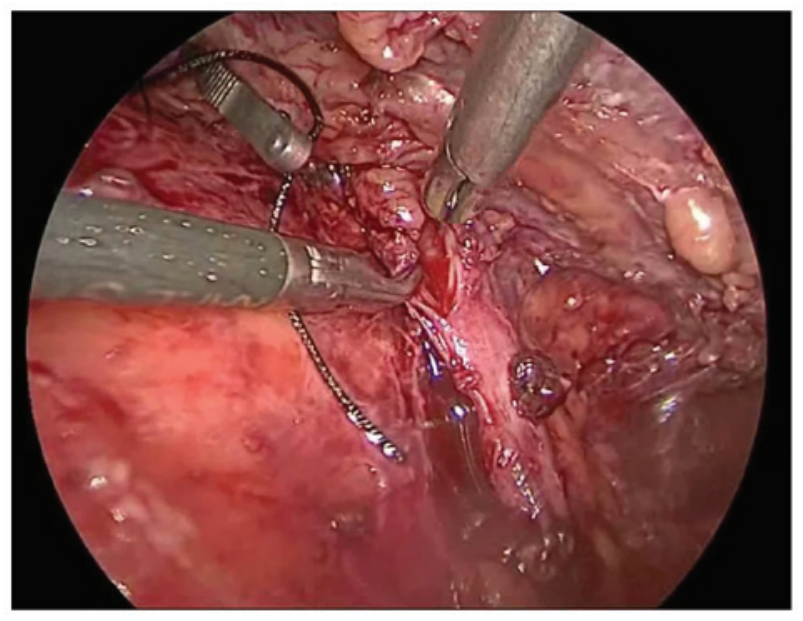

Figure 1. Incision along the direction of ureter to renal pelvis.

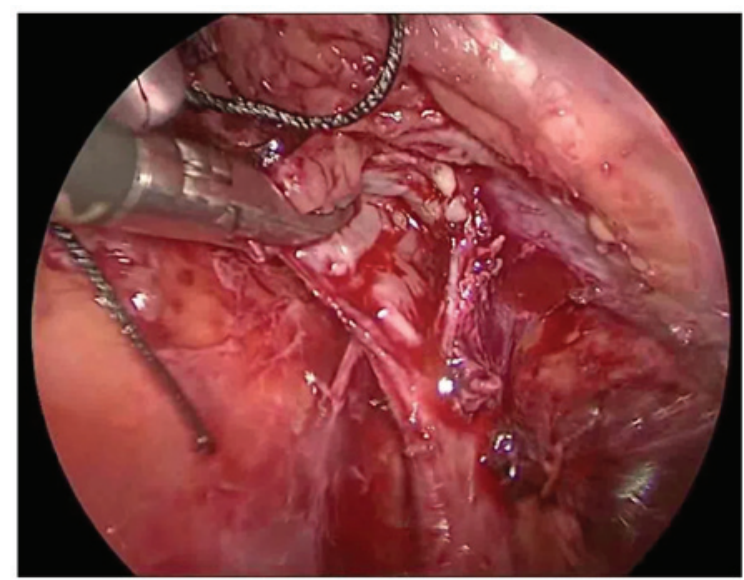

Figure 2. Incision expanded to the junction of the renal parenchyma (posterior lower segment) and pelvis.

volume of drainage fluid, the drainage fluid becoming pale yellow or dramatic high levels of creatinine detected in the drainage fluid. No such corresponding signs were observed in any of the patients. The drainage tube was routinely removed on day 3 after the operation without any indication of urine leakage. For all patients, the double-J stent was removed through cystoscopy at one month after the operation under local anesthesia.

Follow-up. The post-operative laboratory tests (including complete blood count or renal function) were performed on day 3 after the operation for inpatients and 6 months for outpatients. It was required that all patients should stay at the hospital for at least 3 days following surgery. The SFR was evaluated on day 3 after the operation by kidney-ureter-bladder radiologic imaging and at 6 months by US or IVU. All intra- and post-operative complications up to 12 months were recorded and classified according to the Clavien-Dindo grading system $(19,20)$. All procedures were performed by the same experienced surgeon.

Statistical analysis. All analyses were performed using SPSS (version 19.0; IBM Corporation). Values are expressed

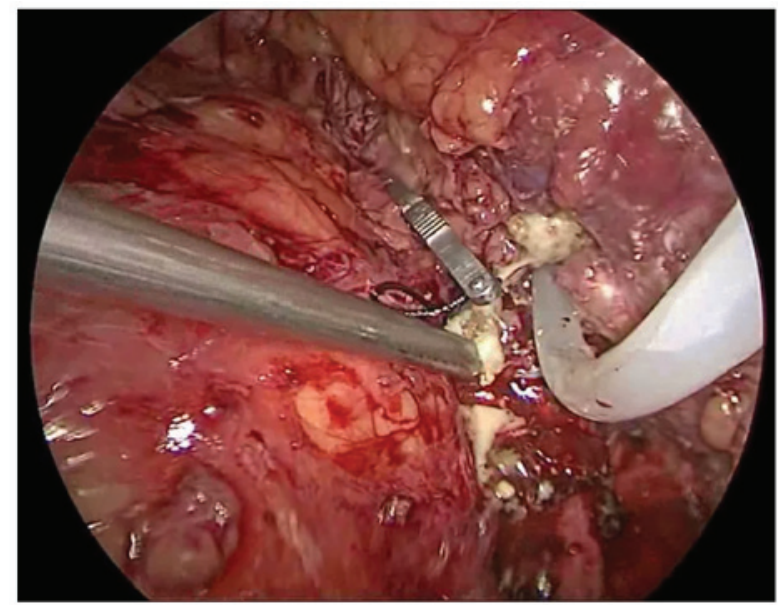

Figure 3. Pediatric catheter was used to flush out residual stones and pus with normal saline.

as the mean \pm standard deviation for continuous variables, and as numbers and percentages for categorical variables. Comparisons were performed using t-tests for normally distributed data (determined via the Shapiro-Wilk test in SPSS). All $\mathrm{P}$-value analyses were two-sided and $\mathrm{P}<0.05$ was considered to indicate a statistically significant difference.

\section{Results}

Demographic and perioperative parameters. All patients (18 females and 10 males) successfully underwent the procedures and there was no conversion to open surgery in any case. The detailed parameters are provided in Tables I and II. The mean age was $57.0 \pm 10.81$ years and the mean stone size (largest diameter) was $3.3 \pm 0.79 \mathrm{~cm}$. The calculi of 20 patients were located in the intrarenal pelvis and those of 8 patients were in the extrarenal pelvis. The mean operation time, warm ischemia time and post-operative hospital stay were 114.4 $\pm 12.09 \mathrm{~min}, 28.1 \pm 4.23 \mathrm{~min}$ and $5.8 \pm 1.42$ days, respectively.

Hemoglobin and serum creatinine levels. The mean hemoglobin drop at day 3 after the operation was $0.5 \pm 0.38 \mathrm{~g} / \mathrm{dl}$ and there was no blood transfusion required in any patient. The change in the serum creatinine levels (pre-operative vs. post-operative day 3) was $6.0 \pm 20.03 \mu \mathrm{mol} / \mathrm{l}$ (t-test $\mathrm{P}=0.53$ ) and $-4.5 \pm 15.13 \mu \mathrm{mol} / 1$ (pre-operative vs. post-operative after 6 months; t-test $\mathrm{P}=0.64)$. While there was no significant difference, it is possible that the renal function was slightly decreased by the influence of surgical procedure in the short-term outcome and increased in the long-term.

Follow-up and complications. The SFR was $100 \%$ as determined at 3 days by kidney-ureter-bladder radiologic imaging and at 6 months by urinary US or IVU. Mild post-operative complications (Grade I or II) were reported in 6 patients, including temporary elevated body temperature and constant elevated body temperature $\left(>38.5^{\circ} \mathrm{C}\right)$. No severe complications appeared, including urine leakage, sepsis, residual stones requiring auxiliary procedures or any circumstance demanding surgical interventions. 
Table I. Demographic and pre-operative characteristics of the patients $(n=28)$.

\begin{tabular}{lc}
\hline Parameter & Value \\
\hline Age (years) & $57.0 \pm 10.81$ (range, 40-74) \\
Sex (female/male) & $18 / 10$ \\
Side (left/right) & $17 / 11$ \\
Intrarenal/extrarenal pelvis & $20 / 8$ \\
Stone size (cm) & $3.3 \pm 0.79$ \\
Urine analysis (WBC/HPF) & $21.5 \pm 10.51$ \\
Positive urine culture & $5(17.9)$ \\
\hline
\end{tabular}

Values are expressed as the mean \pm standard deviation, $\mathrm{n}$ or $\mathrm{n}(\%)$. WBC/HPF, white blood cells per high-power field.

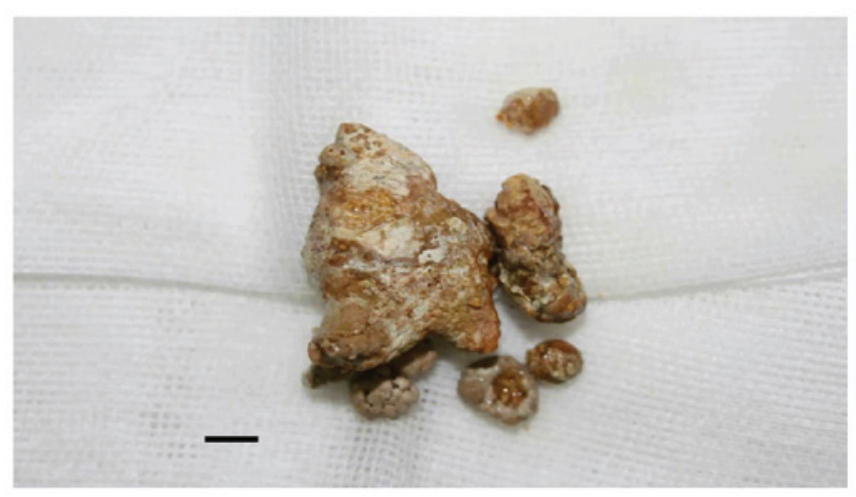

Figure 4. Renal staghorn stones after removal (scale bar, $1 \mathrm{~cm}$ ).

\section{Discussion}

Urinary stone disease, also known as urolithiasis or nephrolithiasis, is an important healthcare problem affecting adults and children $(21,22)$. Technical advances have improved surgical treatment strategies for urolithiasis, including PCNL, extracorporeal shock wave lithotripsy and LP. Staghorn renal stone is a specific type of urolithiasis morphology, which is branched and occupies a large portion of the collecting system (2). Typically, it fills the renal pelvis and branches into several or all of the calyces (2). However, there is no consensus regarding the precise definition of staghorn renal stone and designation of 'partial' or 'complete' staghorn stone does not comprise any specific volume criteria (2). Staghorn renal stone remains a challenge in urology and the goal of treatment is to inhibit recurrence, control infection-associated complications and protect patients' renal function. Tremendous improvements in endourologic procedures have transformed the surgical management of staghorn renal stones (18). According to the AUA and EAU guidelines, which are the most frequently referenced guidelines worldwide, percutaneous nephrolithotomy is the gold standard procedure for management of large renal stone, including staghorn renal stone $(2-4,23)$.

However, treatment for complex, large-burden staghorn stones by PCNL usually requires multiple tracts or multiple sessions. In addition, intra- or post-operative complications of PCNL affect the effectiveness and safety of procedures for
Table II. Peri- and post-operative data for the cohort $(n=28)$.

\begin{tabular}{|c|c|}
\hline Parameter & Value \\
\hline Operation time (min) & $114.4 \pm 12.09$ \\
\hline Warm ischemia time (min) & $28.1 \pm 4.23$ \\
\hline Hemoglobin drop at day $3(\mathrm{~g} / \mathrm{dl})$ & $0.5 \pm 0.38$ \\
\hline \multicolumn{2}{|l|}{ Change in creatinine $(\mu \mathrm{mol} / \mathrm{l})$} \\
\hline Pre- vs. post-operative day 3 & $\begin{array}{c}6.0 \pm 20.03 \\
(\mathrm{t} \text {-test } \mathrm{P}=0.53)\end{array}$ \\
\hline Pre-operative vs. 6 months & $\begin{array}{c}-4.5 \pm 15.13 \\
(\mathrm{t}-\text { test } \mathrm{P}=0.64)\end{array}$ \\
\hline Blood transfusion & $0(0)$ \\
\hline Post-operative hospital stay (days) & $5.8 \pm 1.42$ \\
\hline \multicolumn{2}{|l|}{ Stone-free status } \\
\hline 3 days & $28(100)$ \\
\hline 6 months & $28(100)$ \\
\hline $\begin{array}{l}\text { Post-operative complications } \\
(\text { grade })^{\mathrm{a}}\end{array}$ & 6 \\
\hline I & $\begin{array}{l}5 \text { (17.9 temporary } \\
\text { elevated body temperature) }\end{array}$ \\
\hline II & $\begin{array}{c}1(3.6 \text { constant elevated } \\
\left.\text { body temperature }>38.5^{\circ} \mathrm{C}\right)\end{array}$ \\
\hline IIIa & $0(0)$ \\
\hline $\mathrm{IIIb}$ & $0(0)$ \\
\hline IV & $0(0)$ \\
\hline
\end{tabular}

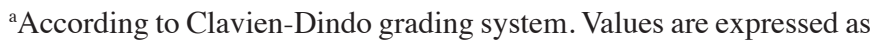
the mean \pm standard deviation or $n(\%)$.

staghorn stones, particularly in patients with concurrent UTI. Despite increased instrument flexibility and miniaturization, post-operative infection-associated complications linked to high intrarenal pelvic pressure, which is at times significantly increased during micro-percutaneous as opposed to conventional PCNL (23), remain life-threatening. Stone recurrence $(31.2 \%)$ and growth $(63.2 \%)$ were reported as challenges in the study by Aminsharifi et al (18) evaluating the long-term outcomes of PCNL monotherapy for staghorn stones in 272 kidneys with a one-session SFR of $76.5 \%$. During these procedures, the increments of intrarenal pelvic pressure was directly associated with complications including postoperative fever and sepsis (24). In a meta-analysis of 14 studies involving 432 patients subjected to LP and 469 receiving PCNL, an equivalent conversion rate, prolonged urine leakage, a higher SFR, and a lower incidence of bleeding, blood transfusion and post-operative fever were identified in the LP group vs. the PCNL group (5). Thus, it appears that LP, RLP and LAN are the optimal treatments for one-session monotherapy for complex staghorn renal stone with concurrent UTI at experienced centers. The patients were fully informed that, according to the EAU and AUA guidelines, the first-line treatment of staghorn stones was PCNL and that WIT would influence the kidney function to a certain extent. Written informed consent was provided by each patient prior to the surgery. It cannot be ignored that patients prefer single-session treatment with high 
SFR and low complications, even though multiple sessions with more minimally invasive treatment may have distinct advantages.

Due to the familiarity of the surgeon with the anatomical structure and care taken to prevent contamination or damage of the abdominal organs, RLP was feasible as a one-session surgical treatment for the patients of the present study. However, in certain patients with large staghorn renal stone, particularly in those with a small neck of the renal calyx, it is difficult to remove all stones by RLP. Violent removal of stones may cause uncontrollable bleeding or even cause damage, which may require nephrectomy. In addition, inadequate exposure of stones and indirect vision may be associated with incomplete clearance of stones. Therefore, depending on the direction and distribution of renal blood vessels, RLP with prolonged renal posterior lower segment incision (small incision prolonged from RLP after temporary blockage of the renal artery) was implemented. The specific incision offers a better exposure and direct vision of stones while avoiding damage of large renal branch vessels.

The renal artery should be completely occluded to provide a clear view of the surgical field. A previous study has demonstrated that selective arterial clamping is sufficient to minimize or avoid bleeding (25). After the renal artery was correctly clamped, it was observed that the kidney became dark, soft and shrunken compared to normal. Laparoscopic experience is crucial for this procedure in order to keep the warm ischemia time in an acceptable range. In the present study, the mean warm ischemia time was $28.1 \pm 4.23 \mathrm{~min}$ and it has been reported that warm ischemia time should not exceed 30 min to maintain the integrity of renal function (25). It has been reported that serum creatinine levels did not significantly increase in patients with a solitary kidney who underwent open anatrophic nephrolithotomy (26). As measured by several types of scintillation technique, post-operative renal function after nephrolithotomy was decreased or increased depending on the different scenarios $(26,27)$. Owing to the limitation of patients' medical insurance, financial situation and personal preference, no scintigraphy examination was performed for measuring renal function pre- or post-operatively, except for those patients with a highly suspected non-functional kidney. Furthermore, the patients preferred one-session monotherapy for treatment due to time and financial reasons. The high one-session SFR of laparoscopic technology is a distinct advantage compared to other treatments. Theoretically, RLP with prolonged renal posterior lower segment incision $(<2 \mathrm{~cm})$ combined with an acceptable warm ischemia time may not significantly decrease the renal function. Furthermore, removal of staghorn stone resolves the potential obstruction and/or infection of the kidney, providing a benefit for renal function. Although there is insufficient evidence, the mean change in serum creatinine levels $(-4.5 \pm 15.13 \mu \mathrm{mol} / \mathrm{l})$ at 6 months post-operatively may suggest an increase in renal function.

Adequate broad-spectrum antibiotic therapy should be provided to patients pre-operatively, since the UTI requires to be controlled at an acceptable level for surgery. In the present study, clinical symptoms, the white blood cell count in urine analysis and the percentage of positive urine culture indicated that these patients had mild to moderate UTIs. It is unfortunate that with its high specificity and low sensitivity, pre-operative urine cultures do not always correlate with renal stone and renal pelvic cultures and remain a poor predictor of infection in the stone or in the upper urinary tract (15). Thus, concurrent UTI was diagnosed according to the different aspects of each individual patient's clinical criteria. UTI (particularly obstructed UTI) may pose an imminent threat to the patient and may induce significant morbidity, including pyonephrosis, sepsis, septic shock and even death (28). At present, patients and urologists desiring a one-session treatment for staghorn renal stone with concomitant infection are facing uncertainties with regard to what the best options are. Of note, according to our experience, RLP with prolonged renal posterior lower segment incision may be an optimal treatment for these patients.

The mean operation and warm ischemia time, post-operative complication rate and long-term SFR of the present study demonstrated the technical feasibility and safety of the procedure. So far, LP, RLP and LAN are all considered as suitable treatments for complex stones with a single-session SFR of up to $>90 \%(5,10,18,29,30)$. Even for large staghorn calculi ( $>4 \mathrm{~cm}$ ), an overall SFR of $~ 90 \%$ was attained in one session (29). Gaur et al (31) first reported the feasibility of RLP for the treatment of staghorn stones in three patients who were considered for open surgery. Singh et al (32) reported on their experience of RLP in 25 patients (including 9 patients with staghorn calculi) and indicated that RLP was a safe and effective procedure for management of staghorn, large and multiple renal calculi. Singal and Dhar (33) compared RLP and open surgery in the management of renal pelvic stone. The large-size study demonstrated that RLP was a promising procedure with acceptable SFR and fewer analgesic requirements, hospital stay and blood loss compared to open surgery. However, this previous study did not focus on patients with staghorn stones in high-risk situations and the regular incision appeared inconvenient for the removal of whole renal caliceal calculi (32). In addition, there is currently no consensus regarding the management of staghorn stones with concurrent UTI. In the patients of the present study, after removing the stone, a pediatric catheter was placed to flush out residual stones and pus with normal saline. According to our experience, it is helpful to increase the single-session SFR and decrease the post-operative infection rate. The extraordinarily high SFR at 6 months may mainly be attributed to the experienced laparoscopic surgeon who performed the operations. It is undeniable that staghorn renal stone remains a health care problem in rural areas of China and the population of patients is large, even though comprehensive health care insurance and routine health examination are gradually reducing the severity of this problem. On the other hand, patient compliance remains low due to the deficient knowledge of the disease and their financial situation. Patients from rural areas of China may be reluctant to receive treatment due to a poor understanding of routine health examinational procedures, a reluctance to treat asymptomatic diseases such as kidney stones, and the inability to afford medical costs. However, as a non-standard management of staghorn stones, the selection of suitable patients should be restricted. Based on our experience, the recommended inclusion criteria are the patient's strong preference for single-session monotherapy considering their financial or medical situation, large or staghorn renal stones (largest diameter $>3 \mathrm{~cm}$ ), patients in 
high-risk situations (e.g. concurrent UTIs) or with complex renal anatomical abnormality and small renal calyx or large stone due to which complete removal of stones is not possible by simple renal pelvis incision; patients meeting all criteria at the same time should be considered as eligible.

The limitation of the present study is the small sample size. Furthermore, comparison with a standard procedure as a reference (PCNL or RLP without prolonged incision) was lacking and should be considered in the future. Due to these drawbacks, it is unclear whether the present procedure is more effective or has less side effects than the currently used standard methods, and it is therefore questionable what the impact of the present procedure actually is.

In conclusion, the present study indicated that RLP with prolonged renal posterior lower segment incision is an effective and safe procedure for patients with staghorn renal stones and concurrent UTI. In the era of minimally invasive surgery, laparoscopic technology still has an irreplaceable role in patients with complex stones under high-risk circumstances. It is a suitable alternative surgical treatment for difficult endourological procedures, particularly as a single-session monotherapy considering the patients' financial and medical situations. In summary, it provides a desirable outcome with minimal morbidity for patients at experienced centers.

\section{Acknowledgements}

Not applicable.

\section{Funding}

No funding was received.

\section{Availability of data and materials}

The datasets used and/or analyzed during the study are available from the corresponding author on reasonable request.

\section{Authors' contributions}

JY performed surgical procedures and supervised the study. BS and XH collected and analyzed the data. BS wrote the manuscript. XH and JY language edited the manuscript.

\section{Ethics approval and consent to participate}

The Ethics Committee of the Sixth People's Hospital Affiliated to Shanghai Jiao Tong University School of Medicine (Shanghai, China) approved the retrospective study and waived written informed consent from the patients. The study protocol is also in accordance with the Declaration of Helsinki.

\section{Patient consent for publication}

Publication of the data in our study does not compromise confidentiality or violate local data protection laws.

\section{Competing interests}

The authors declare that they have no competing interests.

\section{References}

1. Pastore AL, Palleschi G, Silvestri L, Leto A, Ripoli A, Fuschi A, Al Salhi Y, Autieri D, Petrozza V and Carbone A: Combined laparoscopic pyelolithotomy and endoscopic pyelolithotripsy for staghorn calculi: Long-term follow-up results from a case series. Ther Adv Urol 8: 3-8, 2016.

2. Preminger G, Assimos DG, Lingeman JE, Nakada SY, Pearle MS and Wolf JS Jr; AUA Nephrolithiasis Guideline Panel: Chapter 1: AUA guideline on management of staghorn calculi: Diagnosis and treatment recommendations. J Urol 173: 1991-2000, 2005.

3. Assimos D, Krambeck A, Miller NL, Monga M, Murad MH, Nelson CP, Pace KT, Pais VM Jr, Pearle MS, Preminger GM, et al: Surgical management of stones: American Urological Association/Endourological Society Guideline, PART I. J Urol 196: 1153-1160, 2016.

4. Türk C, Petř́ík A, Sarica K, Seitz C, Skolarikos A, Straub M and Knoll T: EAU guidelines on interventional treatment for urolithiasis. Eur Urol 69: 475-482, 2016.

5. Bai Y, Tang Y, Deng L, Wang X, Yang Y, Wang J and Han P: Management of large renal stones: Laparoscopic pyelolithotomy versus percutaneous nephrolithotomy. BMC Urol 17: 75, 2017.

6. Akman T, Binbay M, Sari E, Yuruk E, Tepeler A, Akcay M, Muslumanoglu AY and Tefekli A: Factors affecting bleeding during percutaneous nephrolithotomy: Single surgeon experience. J Endourol 25: 327-333, 2011.

7. Wu H, Zhu S, Yu S, Ding G, Xu J, Li T, Qiao L, Chen Y, Yan J, Cheng X, et al: Early drastic decrease in white blood count can predict uroseptic shock induced by upper urinary tract endoscopic lithotripsy: A translational study. J Urol 193: 2116-2122, 2015.

8. Borofsky MS, Walter D, Shah O, Goldfarb DS, Mues AC and Makarov DV: Surgical decompression is associated with decreased mortality in patients with sepsis and ureteral calculi. J Urol 189: 946-951, 2013 .

9. Li S, Liu TZ, Wang XH, Zeng XT, Zeng G, Yang ZH, Weng H, Meng $\mathrm{Z}$ and Huang JY: Randomized controlled trial comparing retroperitoneal laparoscopic pyelolithotomy versus percutaneous nephrolithotomy for the treatment of large renal pelvic calculi: A pilot study. J Endourol 28: 946-950, 2014.

10. Aminsharifi A, Hadian P and Boveiri K: Laparoscopic anatrophic nephrolithotomy for management of complete staghorn renal stone: Clinical efficacy and intermediate-term functional outcome. J Endourol 27: 573-578, 2013.

11. Nambirajan T, Jeschke S, Albqami N, Abukora F, Leeb $\mathrm{K}$ and Janetschek G: Role of laparoscopy in management of renal stones: Single-center experience and review of literature. J Endourol 19: 353-359, 2005.

12. Singer M, Deutschman CS, Seymour CW, Shankar-Hari M, Annane D, Bauer M, Bellomo R, Bernard GR, Chiche JD, Coopersmith CM, et al: The third international consensus definitions for sepsis and septic shock (Sepsis-3). JAMA 315: 801-810, 2016.

13. Seymour CW, Liu VX, Iwashyna TJ, Brunkhorst FM, Rea TD, Scherag A, Rubenfeld G, Kahn JM, Shankar-Hari M, Singer M, et al: Assessment of clinical criteria for sepsis: For the third international consensus definitions for sepsis and septic shock (Sepsis-3). JAMA 315: 762-774, 2016.

14. Shankar-Hari M, Phillips GS, Levy ML, Seymour CW, Liu VX, Deutschman CS, Angus DC, Rubenfeld GD and Singer M; Sepsis Definitions Task Force: Developing a new definition and assessing new clinical criteria for septic shock: For the third international consensus definitions for sepsis and septic shock (Sepsis-3). JAMA 315: 775-787, 2016.

15. Jiang JT, Li WG, Zhu YP, Sun WL, Zhao W, Ruan Y, Zhong C, Wood K, Wei HB, Xia SJ and Sun XW: Comparison of the clinical efficacy and safety of retroperitoneal laparoscopic ureterolithotomy and ureteroscopic holmium laser lithotripsy in the treatment of obstructive upper ureteral calculi with concurrent urinary tract infections. Lasers Med Sci 31: 915-920, 2016.

16. Chipde SS and Agrawal S: Retroperitoneoscopic pyelolithotomy: A minimally invasive alternative for the management of large renal pelvic stone. Int Braz J Urol 40: 123-124, 2014.

17. Al-Hunayan A, Khalil M,Hassabo M,Hanafi A and Abdul-Halim H: Management of solitary renal pelvic stone: Laparoscopic retroperitoneal pyelolithotomy versus percutaneous nephrolithotomy. J Endourol 25: 975-978, 2011.

18. Aminsharifi A, Irani D, Masoumi M, Goshtasbi B, Aminsharifi A and Mohamadian R: The management of large staghorn renal stones by percutaneous versus laparoscopic versus open nephrolithotomy: A comparative analysis of clinical efficacy and functional outcome. Urolithiasis 44: 551-557, 2016. 
19. Mitropoulos D, Artibani W, Biyani CS, Bjerggaard Jensen J, Rouprêt M and Truss M: Validation of the Clavien-Dindo grading system in urology by the European association of urology guidelines ad hoc panel. Eur Urol Focus 4: 608-613, 2018.

20. Dindo D, Demartines N and Clavien P: Classification of surgical complications: A new proposal with evaluation in a cohort of 6336 patients and results of a survey. Ann Surg 240: 205-213, 2004.

21. Kirkali Z, Rasooly R, Star RA and Rodgers GP: Urinary stone disease: Progress, status, and needs. Urology 86: 651-653, 2015.

22. Clayton DB and Pope JC: The increasing pediatric stone disease problem. Ther Adv Urol 3: 3-12, 2011.

23. Zumstein V, Betschart P, Abt D, Schmid HP, Panje CM and Putora PM: Surgical management of urolithiasis-a systematic analysis of available guidelines. BMC Urol 18: 25, 2018

24. Tepeler A, Akman T, Silay MS, Akcay M, Ersoz C, Kalkan S, Armagan A and Sarica K: Comparison of intrarenal pelvic pressure during micro-percutaneous nephrolithotomy and conventional percutaneous nephrolithotomy. Urolithiasis 42: 275-279, 2014.

25. Giedelman C, Arriaga J, Carmona O, de Andrade R, Banda E, Lopez R, Preminger G and Sotelo RJ: Laparoscopic anatrophic nephrolithotomy: Developments of the technique in the era of minimally invasive surgery. J Endourol 26: 444-450, 2012.

26. Morey AF, Nitahara KS and McAninch JW: Modified anatrophic nephrolithotomy for management of staghorn calculi: Is renal function preserved? J Urol 162: 670-673, 1999.

27. Simforoosh N, Aminsharifi A, Tabibi A, Noor-Alizadeh A, Zand S, Radfar MH and Javaherforooshzadeh A: Laparoscopic anatrophic nephrolithotomy for managing large staghorn calculi. BJU Int 101: 1293-1296, 2008

28. Sammon JD, Ghani KR, Karakiewicz PI, Bhojani N, Ravi P, Sun M, Sukumar S, Trinh VQ, Kowalczyk KJ, Kim SP, et al: Temporal trends, practice patterns, and treatment outcomes for infected upper urinary tract stones in the United States. Eur Urol 64: 85-92, 2013.
29. Gandhi HR, Thomas A, Nair B and Pooleri G: Laparoscopic pyelolithotomy: An emerging tool for complex staghorn nephrolithiasis in high-risk patients. Arab J Urol 13: 139-145, 2015.

30. Simforoosh N, Radfar MH, Nouralizadeh A, Tabibi A, Basiri A, Mohsen Ziaee SA, Sarhangnejad R and Abedinzadeh M: Laparoscopic anatrophic nephrolithotomy for management of staghorn renal calculi. J Laparoendosc Adv Surg Tech A 23: 306-310, 2013

31. Gaur DD, Trivedi S, Prabhudesai MR and Gopichand M: Retroperitoneal laparoscopic pyelolithotomy for staghorn stones. J Laparoendosc Adv Surg Tech A 12: 299-303, 2002.

32. Singh V, Gupta P, Rudramani, Singh SK, Kumar S, Verma S and Srivastava S: Laparoscopic retroperitoneal pyelolithotomy at UP Rural Institute of Medical Sciences and Research: Our initial experience of 25 cases. Int Surg J 3: 488-491, 2016.

33. Singal R and Dhar S: Retroperitoneal laparoscopic pyelolithotomy in renal pelvic stone versus open surgery - a comparative study. Clujul Med 91: 85-91, 2018.

This work is licensed under a Creative Commons Attribution-NonCommercial-NoDerivatives 4.0 International (CC BY-NC-ND 4.0) License. 